Link model simulation and power penalty specification of the versatile link systems

This article has been downloaded from IOPscience. Please scroll down to see the full text article.

2011 JINST 6 C01088

(http://iopscience.iop.org/1748-0221/6/01/C01088)

View the table of contents for this issue, or go to the journal homepage for more

Download details:

IP Address: 137.138.124.233

The article was downloaded on 27/07/2012 at 08:28

Please note that terms and conditions apply. 


\title{
Link model simulation and power penalty specification of the versatile link systems
}

\section{Gong, ${ }^{a}$ C. Liu, ${ }^{a}$ T. Liu, ${ }^{a}$ T. Huffman, ${ }^{b}$ A. Prosser, ${ }^{c}$ J. Troska, ${ }^{d}$ F. Vasey, ${ }^{d}$}

\section{A. Weidberg, ${ }^{b}$ A. Xiang, ${ }^{a, 1}$ J. Ye ${ }^{a}$ and L. Zhu ${ }^{a}$}

\author{
${ }^{a}$ Southern Methodist University, Department of Physics, \\ Dallas, Texas, U.S.A. \\ ${ }^{b}$ Oxford University, Department of Physics, \\ Oxford, U.K. \\ ${ }^{c}$ Fermilab, Electronic Systems Engineering (ESE) Department, \\ Batavia, Illinois, U.S.A. \\ ${ }^{d}$ CERN, Physics and Engineering Department, \\ Geneva, Switzerland \\ E-mail: cxiang@smu.edu
}

\begin{abstract}
This paper presents simulation and experimental studies of optical power penalties on the Versatile Link, a common R\&D project on high-speed optical link for SLHC experiments. The 10 Gigabit Ethernet (10GbE) link model is examined and conservative link power penalties are predicted. We conduct parameter sensitivity analyses and find that the transmitter characteristics affect the link power penalties most. Power penalty differences of multi-mode and single-mode commerical transceiver modules over different fiber lengths are tested to be within the simulation limits. The optical power budgets are then proposed for different Versatile Link variants.
\end{abstract}

KEYWORDS: Optical detector readout concepts; Radiation-hard electronics; Front-end electronics for detector readout

\footnotetext{
${ }^{1}$ Corresponding author.
} 


\section{Contents}

1 Introducion 1

2 Optical link power budget 2

3 Link model and BER test 3

4 Radiation degradation 4

5 Power penalty specification 5

6 Conclusion 6

\section{Introducion}

The Versatile Link project aims to provide a radiation and magnetic field tolerant, high-speed (4.8 Gbps tentative) optical link for the readout and control of SLHC experiments [1]. For a large scale integration project like this, a system level specification is required to ensure the functionality, environmental resistance and operational reliability of the Versatile Link in the proposed applications. One of the most important metrics to be determined is the optical power budget, which specifies a link's signal strengths and power allocations for a given level of performance. On one hand, the distribution of the available power among the link segments should take into account the full range of transmission penalties, the radiation induced degradations as well as a safety margin for unexpected losses. On the other hand, the required optical power levels of the link transceivers should not exceed the manufacturability of these components.

Relevant industrial standards are 10GBASE-SR and 10GBASE-LR as well as fiber channel 400-SM-LC and 400-M5E-SN [2, 3], which have gained international support over the years and to which the commercial components, namely, the back-end, of the Versatile Link will comply. Due to the design philosophy of "worst case" operations and the "interoperability" use of components, optical power budget recommendations by these standards are quite conservative and leave no margins for customization. Since the Versatile Link operates at a lower data rate and a shorter target length (around 150 meters from the front-end detector to the back-end counting room), it is beneficial to re-evaluate the link penalties. More importantly, since the Versatile Link frontend components are expected to suffer significant power degradation from radiation during their lifetimes, and the total losses cannot be accommodated by the existing margins, it is necessary to re-examine the power level limits on the link components. The goal is to propose a conservative yet realistic power budget.

In section 2, the typical Versatile Link transmission scheme and the optical link budget process are described. The IEEE 10GbE link model is investigated in section 3, where bit error rate (BER) 


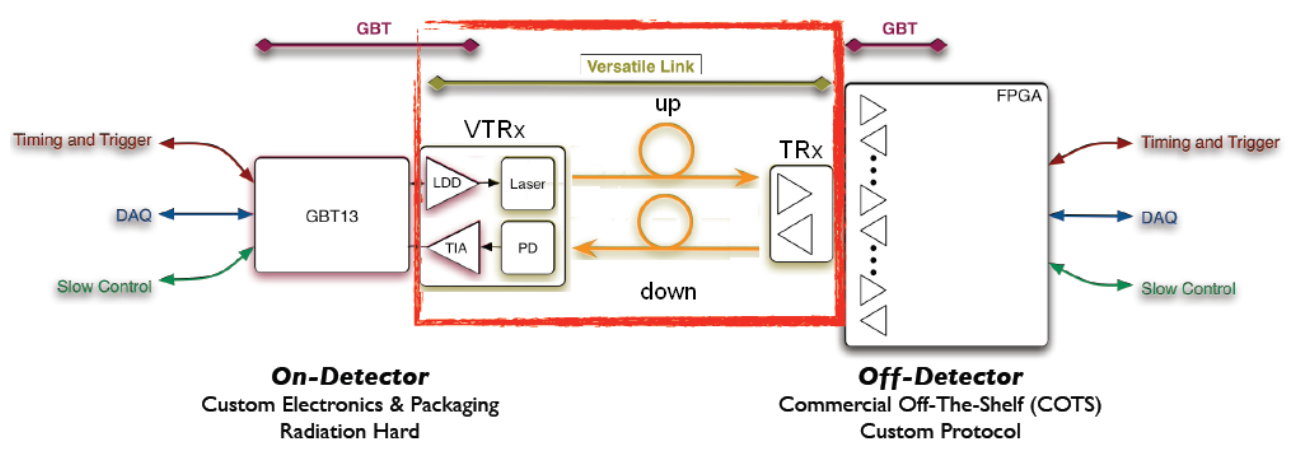

Figure 1. Block diagram of a possible data transmission scheme using the Versatile Link.

measurements on reference links with commercial transceivers are also reported. A crucial part of the power budget is based on the knowledge of device radiation tolerance. This is discussed in section 4. The overall Versatile Link power budget is proposed in section 5 followed by a concluding discussion in section 6 .

\section{Optical link power budget}

The physical layer of a typical optical link comprises the laser-based transmitter circuitry, the PIN photodiode-based receiver circuitry and the fiber medium. A block diagram of a possible transmission scheme deployed by the Versatile Link is shown in figure 1. The central red block encompasses the Versatile Link components. The link is bi-directional and accommodates devices that support both multi-mode operation with a center-wavelength of $850 \mathrm{~nm}$ and single-mode operation with a center-wavelength of $1310 \mathrm{~nm}$.

Two types of electro-optical transceivers are used in the Versatile Link. The on detector module, the versatile transceiver (VTRx), will be custom designed. It consists of a radiation tolerant laser driver (LDD) and a qualified laser diode on the transmitting side; a qualified PIN diode and a radiation tolerant post-amplifier (TIA) on the receiving side. The off detector module, the standard transceiver (TRx), will be procured commercial-off-the-shelf (COTS), as will be the fiber cables.

Given the on and off detector transceiver differences and the two wavelengths of operation, four link variants are specified individually. In the MM_VTx_Rx and the SM_VTx_Rx configurations, data flow from the versatile transmitter on detectors to a standard receiver in the counting room, using multi-mode and single-mode transceiver modules and over multi-mode and singlemode fibers, respectively. In the MM_Tx_VRx and the SM_Tx_VRx configurations, data flow in the opposite direction from standard transmitters in the counting room to the versatile receivers on detector.

To ensure a link BER below $10^{-12}$ at the target data rate and fiber length, across operation temperatures and throughout life cycle, the optical power budget must be carefully planned ahead so that an adequate receiving signal-to-noise ratio is maintained under all conditions. The process is mapped as the allocation of available power among various loss components, as shown in figure 2. The amount of optical power launched into the link is deduced from the difference between the transmitter minimum output, i.e., Tx_OMA_min (OMA — optical modulation am- 


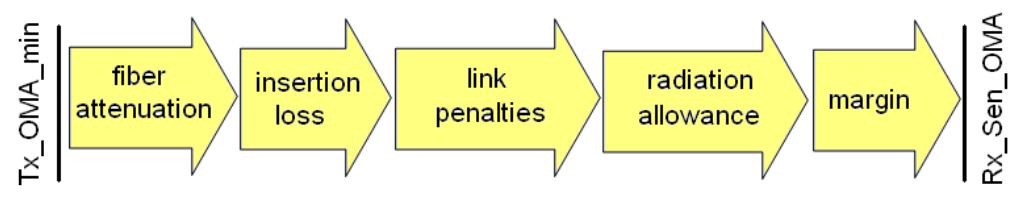

Figure 2. Power budget process in an optical link.

Table 1. Key characteristics in support of the Versatile Link model simulation.

\begin{tabular}{|l|c|c|}
\hline & MM_VL & SM_VL \\
\hline Data rate (Gbps) & 5 & 5 \\
\hline Link length (meter) & 150 & 150 \\
\hline Center wavelength (nm) & 850 & 1310 \\
\hline RMS spectral width (nm) & 0.49 & 0.2 \\
\hline Optical rise/fall time (ps) & 75 & 75 \\
\hline Relative intensity noise (dB/Hz) & -130 & -130 \\
\hline Mode partition noise coefficient & 0.3 & 0 \\
\hline Reflection coefficient & 0 & 0.6 \\
\hline
\end{tabular}

plitude) and the receiver minimum power input, i.e. Rx_sensitivity_OMA. The proposed Versatile Link transceiver power levels are discussed in section 5. Link penalties arising from various mechanisms are discussed in section 3. Since the Versatile Link is designed to meet the requirements of SLHC experiments, allowance must be allocated to radiation degradations, as are described in section 4. Finally, engineering judgment is called for in the assignment of a safety margin.

\section{Link model and BER test}

An open source $10 \mathrm{GbE}$ link model has been developed by the IEEE high speed working group as a tool to facilitate optical physical layer specifications. The model calculates impairments caused by inter-symbol-interference (ISI), mode partition noise (MPN), relative intensity noise (RIN), dutycycle-distortion (DCD), etc. These nonlinear effects result in an increased aggregated link power penalty as the data rate and link length increase.

The link model is populated with key parameter values that represent the Versatile Link operation conditions, as summarized in table 1 . The simulation renders a maximum penalty of $1.0 \mathrm{~dB}$ for the multi-mode versatile link and a maximum penalty of $1.5 \mathrm{~dB}$ for the single-mode versatile link operations. Compared to $4.7 \mathrm{~dB}$ and $3.2 \mathrm{~dB}$ in the 10GBASE-S and 10GBASE-L budgets, the allocation for link penalties are largely reduced since the Versatile Link operates at a lower data rate and shorter reach.

Power penalty dependence on the transmitter, fiber and receiver characteristics was examined and two sets of sensitivity charts are shown in figure 3. We found that in the multi-mode link, power penalty is most sensitive to the optical rise/fall time, $\mathrm{T}_{r / f}(20 \%-80 \%)$, in the sense that the power penalty change is steep in a wide working range. Whereas for the other parameters, i.e., RIN noise, RMS spectral width and receiver bandwidth, the power penalty change is relatively flat in certain working ranges. In the single-mode link, reflection is another sensitive parameter. But the reflection can be controlled through isolators or angle polished connectors to be less than $-12 \mathrm{~dB}$. 


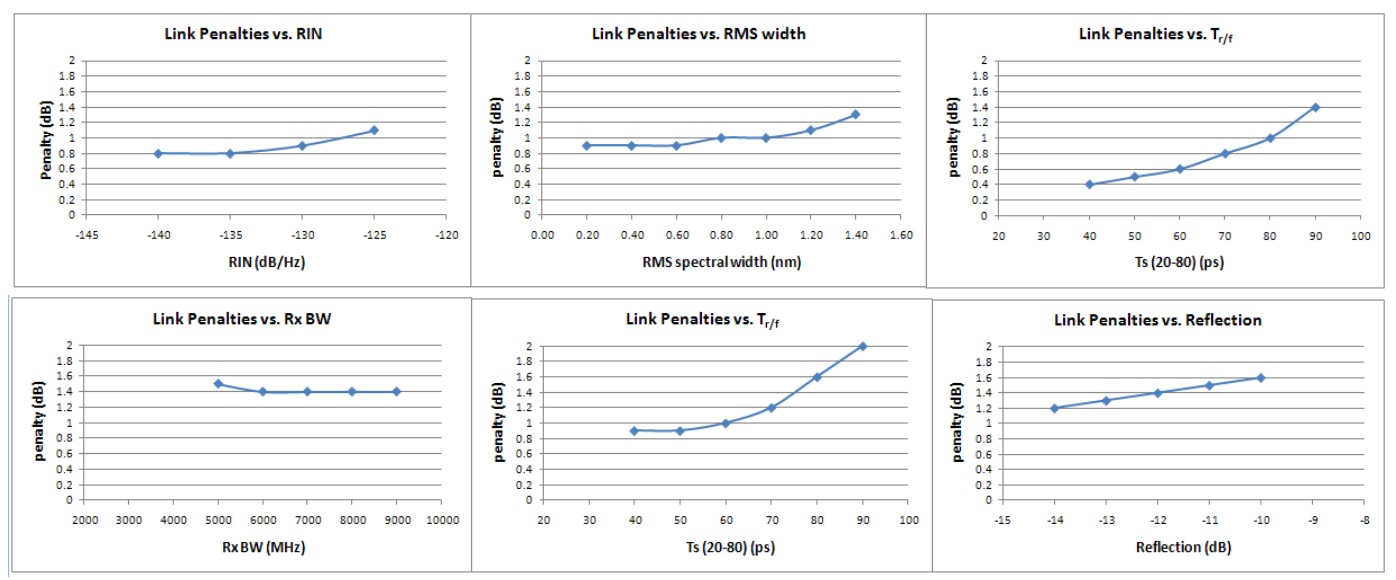

Figure 3. Top: MM_VL link and bottom: SM_VL link characteristic sensitivity charts.
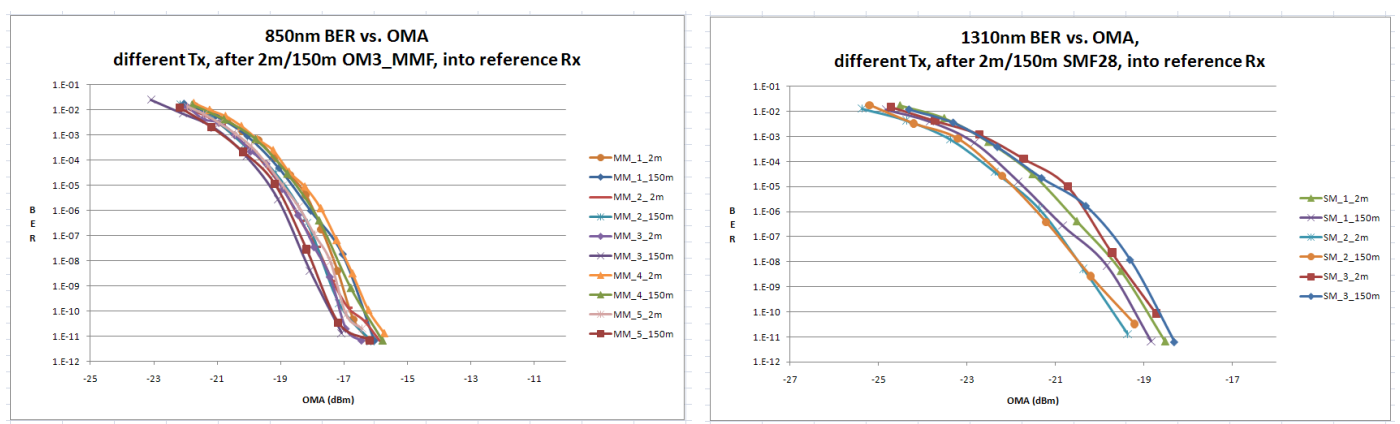

Figure 4. Link power penalties deduced from BER measurements. Left: differences among multi-mode links; right: differences among single-mode links (OMA — optical modulation amplitude measured at the receiver input).

In the previous table, the optical rise/fall time is set to the high end of the target working range to ensure the conservativeness of the link analyzed.

Measurement of link power penalty requires comparison of BER over a typical range since the power penalty is manifested as the horizontal shift in the BER curves. Both multi-mode and single-mode links are constructed using a batch of commercial transceiver modules. A number of different transmitters are launched into different fibers and connected to one reference receiver. The fibers range from 2 meters to 150 meters. Figure 4 shows that power penalty differences among these links are all below $1.5 \mathrm{~dB}$. It further shows that the performance variations of the transmitters have a slightly larger impact on power penalties than the performance and length variations of the fibers at this range. The link model predictions correlate well with these experimental results and are hence adapted in the power budget calculations presented in section 5 .

\section{Radiation degradation}

Qualified Versatile Link front-end components must withstand a level of radiation up to the SLHC level. In addition, their radiation induced power degradation must fit in the allowance allocated in the overall link budget. Candidate laser, PIN, driver, amplifier and fibers are examined in $[5,6]$. 
Table 2. Versatile Link power budget table with 4 variants.

\begin{tabular}{|c|c|c|c|c|}
\hline configuration/parameter & MM_VTx_Rx & MM_Tx_VRx & SM_VTx_Rx & SM_Tx_VRx \\
\hline \hline Transceiver power level & & & & \\
\hline Tx OMA min & $-3.8 \mathrm{dBm}$ & $-2.0 \mathrm{dBm}$ & $-3.2 \mathrm{dBm}$ & $-2.8 \mathrm{dBm}$ \\
\hline Rx Sensitivity OMA & $-11.1 \mathrm{dBm}$ & $-13.1 \mathrm{dBm}$ & $-12.6 \mathrm{dBm}$ & $-15.4 \mathrm{dBm}$ \\
\hline Power budget (Tx - Rx) & $7.3 \mathrm{~dB}$ & $7.3 \mathrm{~dB}$ & $9.4 \mathrm{~dB}$ & $9.4 \mathrm{~dB}$ \\
\hline Fiber attenuation & $0.6 \mathrm{~dB}$ & $0.6 \mathrm{~dB}$ & $0.1 \mathrm{~dB}$ & $0.1 \mathrm{~dB}$ \\
\hline Connection and splice loss & $1.5 \mathrm{~dB}$ & $1.5 \mathrm{~dB}$ & $2.0 \mathrm{~dB}$ & $2.0 \mathrm{~dB}$ \\
\hline Link penalties & $1.0 \mathrm{~dB}$ & $1.0 \mathrm{~dB}$ & $1.5 \mathrm{~dB}$ & $1.5 \mathrm{~dB}$ \\
\hline Tx irradiation degradation & - & - & - & - \\
\hline Rx irradiation degradation & - & $7.0 \mathrm{~dB}$ & - & $9.0 \mathrm{~dB}$ \\
\hline Fiber irradiation degradation & $1.0 \mathrm{~dB}$ & $1.0 \mathrm{~dB}$ & - & - \\
\hline Margin & $3.2 \mathrm{~dB}$ & $0.0 \mathrm{~dB}$ & $5.8 \mathrm{~dB}$ & $0.0 \mathrm{~dB}$ \\
\hline
\end{tabular}

Sources of degradations are described below. Tested or extrapolated to a maximum exposed radiation of $6 \times 10^{15} \mathrm{~cm}^{-2}$ neutron fluence, the corresponding degradation allowances are also allocated.

Both the vertical cavity surface emitting (VCSEL) based multi-mode lasers and the FabryPerot (FP) based single-mode lasers show increased threshold current and decreased slope efficiency. Yet the transmitter power loss should be compensated by increasing drive current and should not exceed $0.05 \mathrm{~dB}$. Both GaAs based multi-mode PIN diodes and InGaAs based singlemode PIN diodes show decreased responsivity, while the InGaAs PIN diodes also suffer from increased leakage current. Overall receiver sensitivity degradations should not exceed $9.6 \mathrm{~dB}$ for GaAs devices and $5.4 \mathrm{~dB}$ for InGaAs devices. PIN diode and post amplifier circuits are also vulnerable to single event upsets (SEU). A customized coding scheme successfully demonstrated the resistance against SEU induced single and burst errors. Different fiber types degrade differently. Radiation induced attenuation is worse at cold temperatures. A few radiation resistant grade candidates are identified and expected to perform most optimistically. Qualified fiber radiation induced attenuation should not exceed $1.0 \mathrm{~dB}$ for multi-mode fibers and $0.05 \mathrm{~dB}$ for single-mode fibers.

\section{Power penalty specification}

Power budget specifications are then proposed for the four variants of the Versatile Link, as listed in table 2. In addition to link penalties and radiation degradations described in the previous sections, fiber attenuation is $3.5 \mathrm{~dB} / \mathrm{km}$ for multi-mode and $0.4 \mathrm{~dB} / \mathrm{km}$ for single-mode. Connector and splice loss is $1.5 \mathrm{~dB}$ for multi-mode and $2.0 \mathrm{~dB}$ for single-mode, which supports 3 or 4 connections with an insertion loss of no more than $0.5 \mathrm{~dB}$ each.

Standard 10GbE compliant transmitter power and receiver sensitivity specifications are applied to the two uplinks, MM_VTx_Rx and SM_VTx_Rx. After the modification on link penalties and the insertion of radiation degradations, these two uplinks meet budget with an increased safety margin. In the two downlinks, MM_Tx_VRx and SM_Tx_VRx, the specifications of the versatile receiver and the corresponding transmitter must exceed those of the standard modules in order to compensate for the large radiation deficits. These elevated power requirements on the downlink transceivers are within technology limits, but mandate individual screening tests. And the safety margin is reduced to zero. 


\section{Conclusion}

Link model simulations and BER tests are performed to evaluate the optical power penalties in the Versatile Link power budget. The results conform well and support a sizable reduction in the allocation for link penalties. However, to accommodate the radiation degradation of the front-end components, power levels of the downlink transceivers still need to be elevated.

Currently there are not enough safety margins to cover environmental and other unexpected losses in the down links. Several further explorations are possible. At the system level, receiver degradation can be relieved by positioning the components further away from detector. At the component level, extra power can be garnered by modest redesign of the laser coupling interface. At the data transmission level, custom coding is known to provide single event upset resistance, which can be quantified as potential power gain.

\section{Acknowledgments}

The authors acknowledge the US-ATLAS R\&D program and the US Department of Energy for funding this work. We would also like to acknowledge Dr. Gary Evans from SMU, Dr. Jim Tatum from Finisar and John Chramowicz from FNAL for beneficial discussions.

\section{References}

[1] F. Vasey et al., The Versatile Link common project, 2003 JINST 4 P12003.

[2] Fiber Channel Physics Interfaces, FC-PI-5, ANSI INCITs.352:2002.

[3] IEEE std 802.3ae. Carrier sense multiple access with collision detection access method and physics layer specification (2008).

[4] IEEE P802.3ae 10Gb/s ethernet task force serial PMD documents, http://www.ieee802.org/3/ae/public/adhoc/serial_pmd/documents/.

[5] T. Huffman et al., The radiation tolerance of specific optical fibres exposed to $650 \mathrm{kGy}$ (Si) of ionizing radiation, 2009 JINST 4 P07010.

[6] J. Troska et al., The versatile transceiver proof of concept, in the proceedongs of the Topical Workshop on Electronics in Particle Physics (TWEPP09), September 21-25, Paris, France (2009). 